\title{
Caracterização óptica e estrutural de ortoferritas de lantânio dopadas com cromo e alumínio
}

\section{(Optical and structural characterization of lanthanum orthoferrites doped with chromium and aluminum)}

\author{
J.L.S.Dutra', G.C.B.Dantas ${ }^{1 *}$, P.M.Pimentel ${ }^{1}$, F.M.Aquino ${ }^{2}$, A. F. Costa ${ }^{3}$, \\ A.C.Lima ${ }^{3}$, R. M. P. B. Oliveira ${ }^{4}$ \\ ${ }^{1}$ Universidade Federal Rural do Semi-Árido, Campus Angicos, 59515-000, Angicos, RN, Brasil \\ ${ }^{2}$ Universidade Federal da Paraíba, João Pessoa, PB, Brasil \\ ${ }^{3}$ Universidade Federal do Rio Grande do Norte, Natal, RN, Brasil \\ ${ }^{4}$ Universidade Federal de Sergipe, São Cristóvão, SE, Brasil
}

\begin{abstract}
Resumo
Esse trabalho teve como objetivo sintetizar óxidos com a estrutura tipo perovskita de composição $\mathrm{LaFeO}_{3}, \mathrm{LaFe}_{0,8} \mathrm{Al}_{0,2} \mathrm{O}_{3} \mathrm{e}$ $\mathrm{LaFe}_{0.8} \mathrm{Cr}_{0,2} \mathrm{O}_{3}$ pelo método sol-gel proteico, visando sua aplicação como pigmento cerâmico. Os pós resultantes do processo de síntese foram calcinados a 600 e $800{ }^{\circ} \mathrm{C}$ e em seguida caracterizados por difração de raios X com refinamento Rietveld, microscopia eletrônica de varredura (MEV), microscopia eletrônica de transmissão (MET), espectroscopia na região do UV-visível e colorimetria CIE-L*a*b*. De acordo com os difratogramas de raios X, todos os pós calcinados foram monofásicos com estrutura tipo perovskita ortorrômbica. As imagens de MEV e MET revelaram que os pós foram porosos e nanométricos. As ferritas de lantânio sem dopagem apresentaram cores em tons pastel, sendo mais claros nos pós dopados com alumínio. Já a ferrita dopada com cromo apresentou coloração marrom claro. As colorações foram mais intensas com o aumento da temperatura de calcinação.

Palavras-chave: pigmento, gelatina, perovskita, ferrita.
\end{abstract}

\section{Abstract}

This work aimed to synthesize oxides with the perovskite structure of $\mathrm{LaFeO}_{3}, \mathrm{LaFe}_{0.8} \mathrm{Al}_{0.2} \mathrm{O}_{3}$ and $\mathrm{LaFe}_{0.8} \mathrm{Cr}_{0.2} \mathrm{O}_{3}$ by proteic sol-gel, in order to use as a ceramic pigment. The resulting powders from the synthesis process were calcined at 600 and $800{ }^{\circ} \mathrm{C}$ and then characterized by X-ray diffraction (XRD) with Rietveld refinement, scanning electron microscopy (SEM), transmission electron microscopy (TEM), UV-visible diffuse reflectance spectroscopy, and CIE-L* $a^{*} b^{*}$ colorimetry. According to the XRD patterns, all calcined powders were single-phase with orthorhombic perovskite structure. The SEM and TEM images revealed that the powders were porous and nanometric. The pure lanthanum ferrites presented pastel colors, being lighter in aluminum-doped powders. The chromium-doped ferrite presented a light brown color. The colors were more intense with increasing calcination temperature.

Keywords: pigment, gelatin, perovskite, ferrite.

\section{INTRODUÇÃO}

A perovskita de formulação $\mathrm{ABO}_{3}$ apresenta estrutura em que os cátions de maior raio iônico, como os metais alcalinos ou lantanídeos, ocupam os sítios A com número de coordenação 12 e os cátion menores, geralmente metais de transição d com número de coordenação 6, ocupam o sítio B [1]. Os óxidos com estrutura tipo perovskita têm sido objetos de interesse da comunidade de pesquisa devido às suas propriedades físicas e químicas. Mais especificamente, as ortoferritas de lantânio possuem um campo de aplicabilidade vasto, tais como óxidos de células a combustível [2,3], catalisadores [4], separadores de gás [5], magnetorresistência gigante negativa [6] e sensores de

*gerbeson_dantas@hotmail.com gás [7, 8], e propriedades magnéticas interessantes, como antiferromagnetismo. Atualmente, esse material devido à sua alta superfície de contato e aliado à forte propriedade óptica do ferro está se tornando objeto de estudo da indústria cerâmica por possuir potencial de pigmento cerâmico. Somando-se a isso, as ortoferritas quando dopadas podem modificar ou potencializar suas propriedades em função das substituições parciais do íon central $\mathrm{Fe}^{3+}$ por outros elementos químicos com propriedades diversas. Além das dopagens, as propriedades podem ser afetadas em função da rota de síntese utilizada para obtenção da mesma.

A ortoferrita de lantânio, segundo a literatura, tem sido obtida por vários métodos, como mecânico-químico $[9,10]$, reação no estado sólido [11], tratamento hidrotérmico [12], sol-gel [13, 14], coprecipitação $[15,16]$, método complexo polimerizável [17], método de sais fundidos [18] e síntese de 
combustão [19]. Recentemente, um método vem chamando atenção dos pesquisadores por sintetizar pós nanométricos em relativamente baixas temperaturas, de maneira rápida e simplificada, cujo princípio consiste em utilizar a gelatina como direcionador orgânico, em substituição ao ácido cítrico e etilenoglicol do método Pechini [20,21]. Nesse sentido, o presente trabalho tem como objetivo sintetizar óxidos com estrutura tipo perovskita de composição $\mathrm{LaFeO}_{3}(\mathrm{LF})$, $\mathrm{LaFe}_{0,8} \mathrm{Al}_{0,2} \mathrm{O}_{3}$ (LFA) e $\mathrm{LaFe}_{0,8} \mathrm{Cr}_{0,2} \mathrm{O}_{3}$ (LFC) pelo método sol-gel proteico, visando sua aplicação como pigmento cerâmico, estudando a sua microestrutura, as propriedades ópticas e a rota de síntese.

\section{MATERIAIS E MÉTODOS}

Os reagentes utilizados para síntese dos pós foram: nitrato de alumínio nona-hidratado $\mathrm{Al}\left(\mathrm{NO}_{3}\right)_{3} \cdot 9 \mathrm{H}_{2} \mathrm{O}$ (Isofar, $99,7 \%)$, nitrato de lantânio hexa-hidratado $\mathrm{La}\left(\mathrm{NO}_{3}\right)_{3} \cdot 6 \mathrm{H}_{2} \mathrm{O}$ (Sigma-Aldrich, 99,9\%), nitrato de ferro (III) nona-hidratado $\mathrm{Fe}\left(\mathrm{NO}_{3}\right)_{3} .9 \mathrm{H}_{2} \mathrm{O}$ (Sigma-Aldrich, $98,9 \%$ ) e nitrato de cromo nona-hidratado $\mathrm{Cr}\left(\mathrm{NO}_{3}\right)_{3} \cdot 9 \mathrm{H}_{2} \mathrm{O}$ (Vetec, $99,9 \%$ ). A gelatina foi cedida pela empresa Gelita.

Os pós foram preparados pelo método sol-gel proteico [20,21]. Esse método consistiu em adicionar água, gelatina e nitratos metálicos em um béquer, sob agitação a $70{ }^{\circ} \mathrm{C}$. $\mathrm{O}$ resultado desta reação foi um gel polimérico (resina) que foi calcinado a $400{ }^{\circ} \mathrm{C}$ para eliminação da matéria orgânica inserida no processo de síntese. Em seguida, os pós precursores foram calcinados por $4 \mathrm{~h}$ a 600 e $800{ }^{\circ} \mathrm{C}$ para obtenção da fase perovskita.

Os pós sintetizados nas duas temperaturas foram caracterizados por meio das técnicas de difração de raios X (DRX) usando um difratômetro (Shimadzu, XRD-6000), seguido de refinamento Rieveld, microscopia eletrônica de varredura (MEV) usando um microscópio (Jeol, SEM LV), microscopia eletrônica de transmissão (MET, Jeol, JEM 3010 URP), colorimetria (Gretag Macbeth, Color-Eye 2180) e espectrometria na região do UV-visível com um espectrofotômetro (Shimadzu, UV-Visible) com reflectância acessória (UV-2550) com comprimento de onda na região de $200-900 \mathrm{~nm}$.

\section{RESULTADOS E DISCUSSÃO}

Os difratogramas de raios $\mathrm{X}$ das amostras $\mathrm{LaFeO}_{3}(\mathrm{LF})$, $\mathrm{LaFe}_{0,8} \mathrm{Al}_{0,2} \mathrm{O}_{3}$ (LFA) e $\mathrm{LaFe}_{0,8} \mathrm{Cr}_{0,2} \mathrm{O}_{3}$ (LFC) tratadas a 600 e $800{ }^{\circ} \mathrm{C}$ por 4 h estão ilustrados na Fig. 1. A identificação das fases deu-se por meio do refinamento Rietveld [22] e o ajuste do padrão foi feito com a ajuda do software MAUD [23]. O refinamento Rietveld revelou que os pós sintetizados apresentaram estrutura tipo perovskita, com simetria ortorrômbica, grupo espacial Pnma, conforme constatada pelo arquivo ICSD 84941. A proximidade entre os difratogramas das amostras LF, LFA e LFC revelou que a substituição parcial do íon $\mathrm{Fe}^{3+}$ pelos íons $\mathrm{Cr}^{3+} \mathrm{e} \mathrm{Al}^{3+}$ foi bem-sucedida, incorporando completamente no sítio $\mathrm{B}$ da estrutura perovskita. Os pós sintetizados pelo método sol-
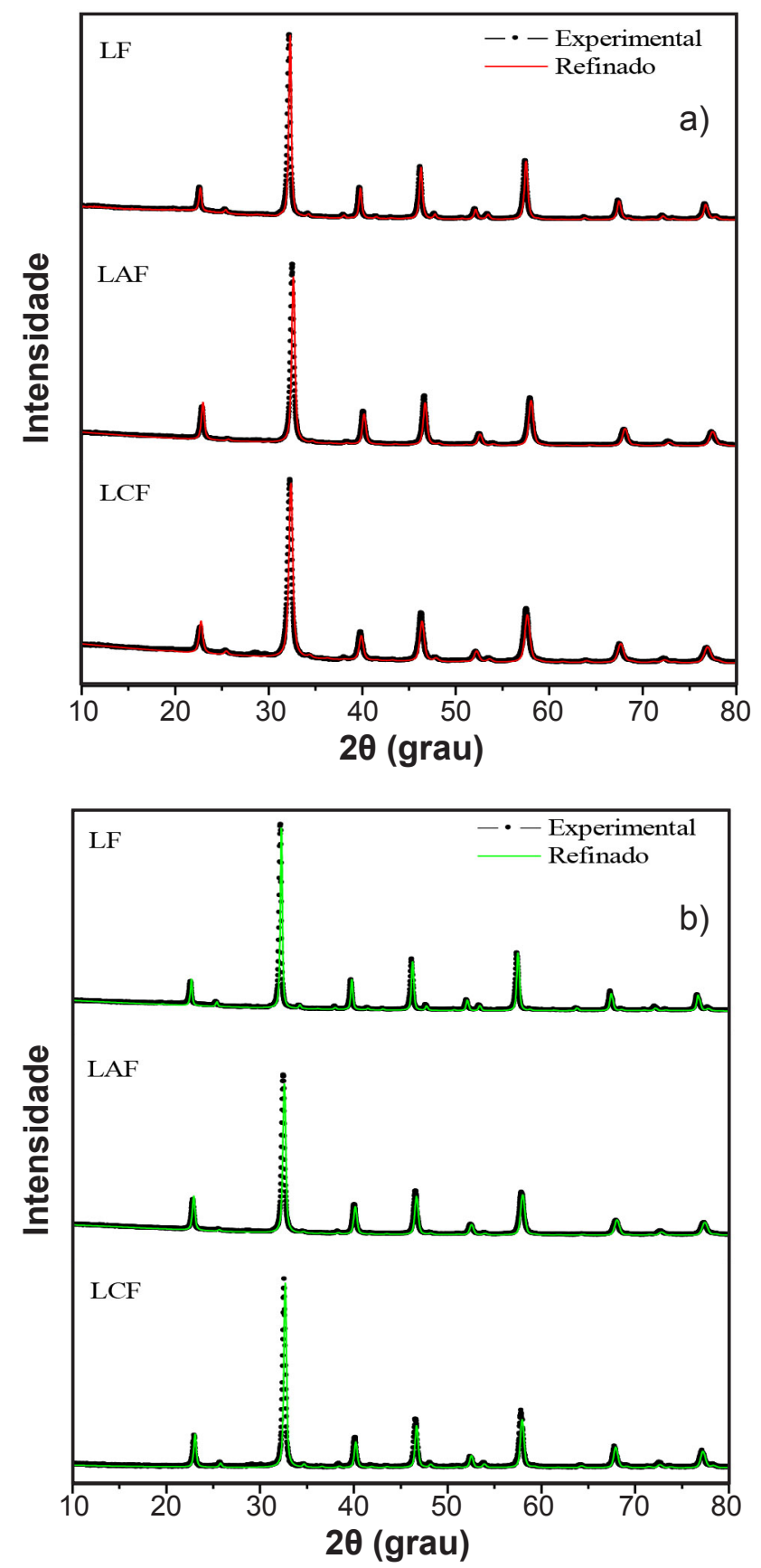

Figura 1: Difratogramas de raios $\mathrm{X}$ das perovskitas LF, LFA e LFC calcinadas a: (a) $600{ }^{\circ} \mathrm{C}$; e (b) $800{ }^{\circ} \mathrm{C}$.

[Figure 1: X-ray diffraction patterns of LF, LFA and LFC perovskites calcined at: (a) $600{ }^{\circ} \mathrm{C}$; and (b) $800^{\circ} \mathrm{C}$.]

gel proteico foram monofásicos em ambas as temperaturas de calcinação, mostrando-se vantajoso na obtenção de ferritas de lantânio e, portanto, alternativo aos métodos convencionais de síntese. A reflexão característica do pico principal da estrutura perovskita aparece no intervalo entre $30^{\circ}<2 \theta<35^{\circ}$ nos difratogramas de todas as amostras, com estreitamento da reflexão conforme aumento da temperatura, o que é um indicativo do aumento da cristalinidade. Resultados similares foram encontrados em outro trabalho [24]. Na literatura, vários autores sintetizaram LF por vários 
métodos: em um dos trabalhos, foi sintetizada a perovskita pelo método sol-gel, mas somente a $1200{ }^{\circ} \mathrm{C}$ foi obtida a fase sem vestígios de fase secundária [25]; enquanto em outro [26], foi obtida LF monofásica pelo método Pechini, porém somente em $900{ }^{\circ} \mathrm{C}$. Tais resultados enfatizam o caráter vantajoso do método sol-gel proteico em relação às outras rotas tradicionais para obtenção de perovskitas monofásicas. O interesse por pós monofásicos se dá pela necessidade dos pigmentos em apresentar homogeneidade de suas propriedades, agregando maior valor tecnológico e econômico ao esmalte produzido.

Os parâmetros microestruturais são mostrados na Tabela I. Observou-se que o tamanho médio do cristalito aumentou conforme a temperatura de calcinação foi elevada, variando entre 58 a $80 \mathrm{~nm}$ para o LF, 43 a $55 \mathrm{~nm}$ para LFA e 30 a $54 \mathrm{~nm}$ para LFC. O aumento do tamanho do cristalito deve-se ao aumento da temperatura, uma vez que a mesma atua como força motriz de coalescência e crescimento das partículas [20]. O valor de otimização do refinamento (SIG) foi aceitável, denotando precisão do refinamento Rietveld, dado que é considerado um bom refinamento quando o valor do SIG é inferior a 2 [20,21].

Imagens de MEV e MET da ferrita de lantânio calcinada a $800^{\circ} \mathrm{C}$ podem ser vistas nas Figs. $2 \mathrm{a}$ e $2 \mathrm{~b}$, respectivamente. A morfologia porosa, observada na imagem de $\mathrm{MEV}$, deve-se à evolução dos gases decorrente do processo de decomposição da gelatina. Aglomerados de partículas $(<200$ $\mathrm{nm}$ ) podem ser claramente vistos nessa imagem, os quais consistem em um grande número de partículas esféricas mantidas juntas por forças interfaciais. Na imagem de MET observa-se que as partículas têm a forma arredondada com dimensões inferiores a $200 \mathrm{~nm}$.

Os espectros de reflectância difusa das perovskitas LF, LFA e LFC calcinadas a 600 e $800^{\circ} \mathrm{C}$ são apresentados na Fig. 3. Os espectros das amostras LF e LFA são bastante semelhantes, uma vez que o íon cromóforo é o $\mathrm{Fe}^{3+} \mathrm{e}$ a banda na região em torno de $700 \mathrm{~nm}$ é atribuída à transição ${ }^{6} \mathrm{~A}_{1 \mathrm{~g}}{ }^{-4} \mathrm{~T}_{2 \mathrm{~g}}$ desse íon em sítio octaédrico [27-29]. Já no óxido LFC, com a incorporação do $\mathrm{Cr}^{3+}$ na estrutura, ocorreu uma sobreposição das bandas dos íons cromóforos $\mathrm{Cr}^{3+} \mathrm{eFe}^{3+}$, de modo que ambos contribuíram para a cor final, resultando em marrom claro. A banda em torno de $570 \mathrm{~nm}$ foi atribuída à transição ${ }^{4} \mathrm{~A}_{2 \mathrm{~g}}\left({ }^{4} \mathrm{~F}\right) \rightarrow{ }^{4} \mathrm{~T}_{2 \mathrm{~g}}\left({ }^{4} \mathrm{~F}\right)$ do íon $\mathrm{Cr}^{3+}$ em sítio octaédrico [30]. As coordenadas colorimétricas no sistema CIE-L*a*b* são mostradas na Tabela II. As coordenadas corroboram com o que pode ser visto no espectro de reflectância (Fig. 3). À medida que a temperatura de calcinação aumentou, a luminosidade $\mathrm{L}$ foi reduzida e, consequentemente, a cor foi um pouco mais escura nos pós calcinados em temperaturas mais altas. Este comportamento ocorreu em função de

Tabela I - Parâmetros microestruturais das perovskitas LF, LFA e LFC.

[Table I - Microstructural parameters of LF, LFA and LFC perovskites.]

\begin{tabular}{cccccccc}
\hline \multirow{2}{*}{ Amostra } & $\begin{array}{c}\text { Temperatura } \\
\left({ }^{\circ} \mathrm{C}\right)\end{array}$ & \multicolumn{2}{c}{$\begin{array}{c}\text { Parâmetro de rede }(\AA) \\
\mathrm{a}(\AA)\end{array}$} & $\mathrm{c}(\AA)$ & $\begin{array}{c}\text { Volume da célula } \\
\text { unitária }(\AA)\end{array}$ & $\begin{array}{c}\text { Tamanho do } \\
\text { cristalito }(\mathrm{nm})\end{array}$ & \multirow{2}{*}{ SIG } \\
\hline \multirow{2}{*}{ LF } & 600 & 5,563 & 7,852 & 5,556 & 242,690 & 58 & 1,39 \\
& 800 & 5,564 & 7,855 & 5,555 & 242,782 & 80 & 1,37 \\
\multirow{2}{*}{ LFA } & 600 & 5,519 & 7,804 & 5,533 & 238,281 & 43 & 1,61 \\
& 800 & 5,524 & 7,810 & 5,523 & 238,276 & 51 & 1,64 \\
\multirow{2}{*}{ LFC } & 600 & 5,554 & 5,549 & 7,843 & 241,715 & 30 & 1,62 \\
& 800 & 5,551 & 5,551 & 7,849 & 241,856 & 54 & 1,65 \\
\hline
\end{tabular}
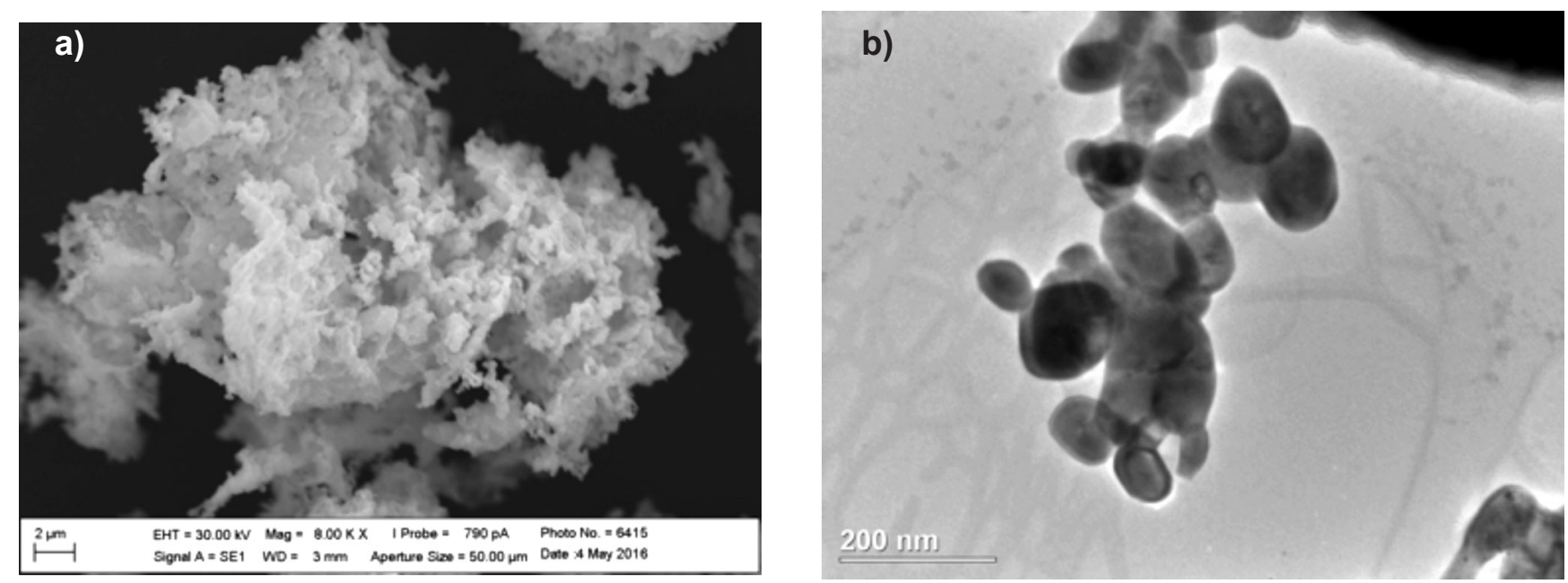

Figura 2: Micrografias de MEV (a) e de MET (b) da LF calcinada a $800{ }^{\circ} \mathrm{C}$.

[Figure 2: SEM (a) and TEM (b) micrographs of LF calcined at $800^{\circ} \mathrm{C}$.] 

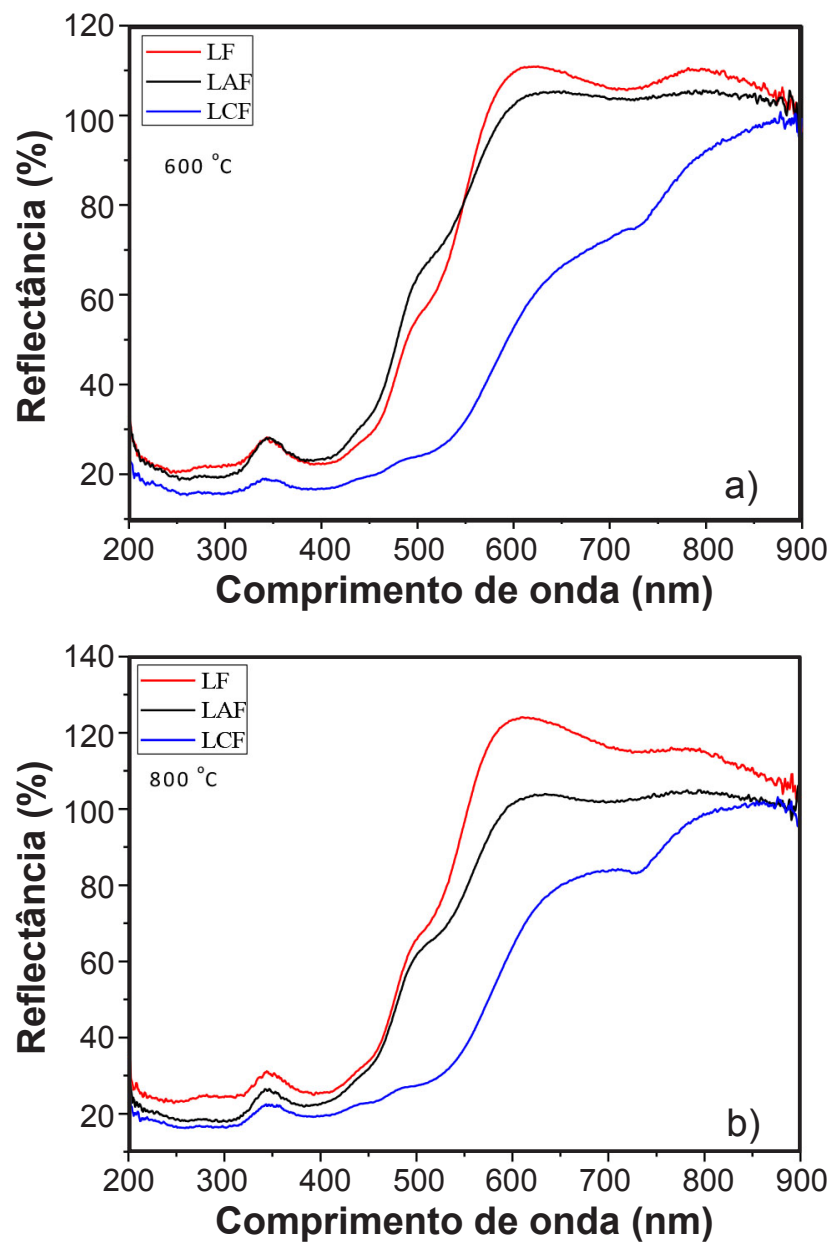

Figura 3: Espectros de reflectância das perovskitas LF, LFA e LFC calcinadas a: (a) $600{ }^{\circ} \mathrm{C}$; e (b) $800{ }^{\circ} \mathrm{C}$.

[Figure 3: Reflectance spectra of LF, LFA and LFC perovskites calcined at: (a) $600{ }^{\circ} \mathrm{C}$; and (b) $800^{\circ} \mathrm{C}$.]

Tabela II - Parâmetros colorimétricos das perovskitas LF, LFA e LFC calcinadas a 600 e $800{ }^{\circ} \mathrm{C}$.

[Table II - Colorimetric parameters of LF, LFA and LFC perovskites calcined at 600 and $800^{\circ} \mathrm{C}$.]

\begin{tabular}{ccccc}
\hline Amostra & Temperatura & $\mathrm{L}^{*}$ & $\mathrm{a}^{*}$ & $\mathrm{~b}^{*}$ \\
\hline \multirow{2}{*}{$\mathrm{LF}$} & $600{ }^{\circ} \mathrm{C}$ & 59,22 & 7,03 & 24,79 \\
& $800^{\circ} \mathrm{C}$ & 58,63 & 6,21 & 26,39 \\
\multirow{2}{*}{ LFA } & $600{ }^{\circ} \mathrm{C}$ & 64,02 & 4,09 & 21,56 \\
& $800^{\circ} \mathrm{C}$ & 58,96 & 3,61 & 25,17 \\
\multirow{2}{*}{ LFC } & $600{ }^{\circ} \mathrm{C}$ & 54,01 & 7,43 & 11,46 \\
& $800{ }^{\circ} \mathrm{C}$ & 53,65 & 14,38 & 13,01 \\
\hline
\end{tabular}

sua microestrutura, uma vez que com o aumento da temperatura de calcinação houve o crescimento do tamanho do cristalito (Tabela I), então a área superficial de contato entre a partícula de pó com o iluminante do colorímetro foi reduzida e, portanto, a superfície de reflexão foi diminuída, resultando em um deslocamento do $\mathrm{L}$ para o preto [21]. Observou-se que no pó LFA, a introdução do $\mathrm{Al}^{3+}$ reduziu a quantidade de cromóforo $\mathrm{Fe}^{3+}$, aumentando a luminosidade
$\mathrm{L}$ e reduzindo os valores de a e b, tornando a cor menos intensa. A substituição do íon $\mathrm{Fe}^{3+}$ por $\mathrm{Cr}^{3+}$ na amostra LFC diminuiu o valor da coordenada $\mathrm{L}$ e aumentou a coordenada $a^{*}$ em relação a $\mathrm{LF}$, tornando o pigmento ligeiramente mais escuro e aumentando a contribuição do componente vermelho para cor.

\section{CONCLUSÕES}

Este trabalho apontou que o método sol-gel proteico foi eficiente em sintetizar pós de $\mathrm{LaFeO}_{3}(\mathrm{LF}), \mathrm{LaFe}_{0,8} \mathrm{Al}_{0,2} \mathrm{O}_{3}$ (LFC) e $\mathrm{LaFe}_{0,8} \mathrm{Cr}_{0,2} \mathrm{O}_{3}$ (LFA) nanométricos e monofásicos e eficaz em função do método ser simples, rápido e econômico em comparação aos outros métodos. Em razão disto, apresenta-se capaz de ser replicado na indústria. Quanto à substituição parcial de $\mathrm{Fe}^{3+}$ por $\mathrm{Al}^{3+}$ e $\mathrm{Cr}^{3+}$, não foi identificada nenhuma alteração significativa na estrutura cristalina da perovskita, conforme corroborado pelos difratogramas de raios $\mathrm{X}$. Quanto à cor, as amostras LF e LFA apresentaram tonalidade pastel, sendo mais clara com a substituição parcial do $\mathrm{Fe}^{3+}$ por $\mathrm{Al}^{3+}$. Já o pó LFC apresentou coloração marrom claro devido à superposição das bandas dos íons cromóforos $\mathrm{Fe}^{3+}$ e $\mathrm{Cr}^{3+}$ contribuindo ambos para a cor final do pigmento.

\section{AGRADECIMENTOS}

Os autores agradecem ao CNPq pelo suporte financeiro e aos laboratórios da UFPB e LNLS pelas análises.

\section{REFERÊNCIAS}

[1] H. Tanaka, M. Misono, Curr. Opin. Solid State Mater. Sci. 5, 5 (2001) 381.

[2] C.S. Cheng, L. Zhang, Y.J. Zhang, S.P. Jiang, Solid State Ionics 179 (2008) 282.

[3] S. Pathak, J. Kuebler, A. Payzant, N. Orlovskaya, J. Power Sources 195 (2010) 3612.

[4] N. Russo, D. Fino, G. Saracco, V. Specchia, Catal. Today 137 (2008) 306.

[5] M.B. Bellakki, C. Madhu, T. Greindl, S. Kohli, P. McCurdy, V. Manivannan, Rare Metals 29 (2010) 491.

[6] D. Hammer, J. Wu, C. Leighton, Phys. Rev. B 69 (2004) 134407.

[7] E. Traversa, S. Matsushima, G. Okada, Y. Sadaoka, Y. Sakai, K. Watanabe, Sens. Actuators, B 25 (1995) 661.

[8] D. Wang, X.F. Chu, M.L. Gong, Nanotechnology 17 (2006) 5501.

[9] Q.W. Zhang, F. Saito, J. Mater. Sci. 36 (2001) 2287.

[10] A.A. Cristóbala, P.M. Botta, P.G. Bercoff, J.M. Porto López, Mater. Res. Bull. 44 (2009) 1036.

[11] S. Acharya, J. Mondal, S. Ghosh, S.K. Roy, P.K. Chakrabarti, Mater. Lett. 64 (2010) 415.

[12] W.J. Zheng, R.H. Liu, D.K. Peng, G.Y. Meng, Mater. Lett. 43 (2000) 19.

[13] X.W. Qi, J. Zhou, Z.X. Yue, Z.L. Gui, L.T. Li, Ceram. Int. 29 (2003) 347. 
[14] G. Shabbira, A.H. Qureshib, K. Saeed, Mater. Lett. 60 (2006) 3706.

[15] P.V. Gosavi, R.B. Biniwale, Mater. Chem. Phys. 119 (2010) 324.

[16] S. Nakayama, J. Mater. Sci. 36 (2001) 5643.

[17] M. Popa, J. Frantti, M. Kakihana, Solid State Ionics 154-155 (2002) 135.

[18] J. Yang, R.S. Li, J.Y. Zhou, X.C. Li, Y.M. Zhang, Y.L. Long, Y.W. Li, J. Alloys Compd. 508 (2010) 301.

[19] H. Shen, G.F. Cheng, A.H. Wu, J.Y. Xu, J.T. Zhao, Phys. Status Solidi A 206 (2009) 1420.

[20] F.S. Oliveira, P.M. Pimentel, R.M.P.B. Oliveira, D.M.A. Melo, M.A.F. Melo, Mater. Lett. 64 (2010) 2700.

[21] P.M. Pimentel, S.V.M. Lima, A.F. Costa, M.S.C. Câmara, J.D.C. Carregosa, R.M.P.B. Oliveira, Ceram. Int. 43 (2017) 6592.

[22] H.M. Rietveld, J. Appl. Crystallogr. 2 (1969) 65.

[23] L. Lutterotti, MAUD, v.2.046 (2006), www.ing.unitn. it/wmaud/.

[24] H. Xu, X. Hu, L. Zhang, Cryst. Growth Des. 8, 7 (2008) 2061.

[25] E. Cao, Y. Qin, T. Cui, L. Sun, W. Hao, Y. Zhang, Ceram. Int. 43 (2017) 7922.

[26] J.D. Cunha, D.M.A. Melo, A.E. Martinelli, M.A.F. Melo, I. Maia, S.D. Cunha, Dyes Pigments 65 (2005) 11.

[27] L.S. Kumari, P.P. Rao, M.L. Reddy, J. Alloy Compd. 461 (2008) 509.

[28] N. Pailhe, A. Wattiaux, M. Gaudon, A. Demourgues, J. Solid State Chem. 181 (2008) 1040.

[29] X.H. Wang, J.G. Li, H. Kamiyama, M. Katada, N. Ohashi, Y. Moriyoshi, T. Ishigak, J. Am. Chem. Soc. 127 (2005) 10982.

[30] P.M. Pimentel, K.M.B. Ferreira, D.K.S. Gomes, A.F. Costa, D.M.A. Mello, R.M.P.B. Oliveira, Mater. Sci. Forum 881 (2016) 7.

(Rec. 11/12/2017, Rev. 31/01/2018, Ac. 14/02/2018) 